\title{
Estudio De La Brecha Empresario-Investigador: La Percepción Del Empresario
}

\author{
Carlos Ángel Méndez-Peón, M.C.
}

Universidad Popular Autónoma del Estado de Puebla, Universidad de Sonora Candidato a doctor en Planeación Estratégica y Dirección de Tecnología

\section{Margarita Herrera-Avilés, PhD}

Instituto Tecnológico y de Estudios Superiores de Monterrey, campus Puebla

\author{
Alfredo Toriz-Palacios, PhD \\ Manuel González-Pérez, PhD, SNI I
}

Universidad Popular Autónoma del Estado de Puebla

doi: 10.19044/esj.2017.v13n10p280 URL:http://dx.doi.org/10.19044/esj.2017.v13n10p280

\begin{abstract}
Competitiveness and productivity are crucial for the development of a country. A more competitive economy can grow faster over time. Technological innovation is the strongest pillar that guarantees long term competitiveness and quality of life for both developed and emerging countries. The University-Enterprise relationship (RUE) is a resource that is used to build a strong pillar of innovation. Therefore, it is important that a good relationship should be established between the main participants, the entrepreneur and the researcher (E-I). However, current literature has largely ignored the problem of the personnel involved. In contributing to the knowledge of this subject, we examined the gap that exists between the entrepreneur and the researcher (ERG), the importance of empathy in the E-I collaboration, and the factors that impact this collaboration the most from the entrepreneur stand point. The study was exploratory and transversal and data was gathered through interviews and questionnaires. The sample consisted of expert entrepreneurs with experience in innovation projects carried out in collaboration with researchers. The results show that $70 \%$ of the interviewed entrepreneurs recognize the existence of ERG. 87.5\% of the entrepreneurs surveyed see empathy as a very significant factor for the E-I relationship, and there were 32 factors that are most influential on this collaboration.
\end{abstract}

Keywords: The Entrepreneur Researcher Gap (ERG), Technological Innovation, University-Enterprise Relationship (RUE), Competitiveness, Strategy, R \& D, Transfer of Technology and Knowledge (TTK) 


\section{Resumen}

La competitividad y la productividad son cruciales para el desarrollo de un país. Una economía más competitiva puede crecer más rápidamente con el tiempo. La innovación tecnológica es el pilar más sólido para asegurar la competitividad a largo plazo y la calidad de vida de los países desarrollados y emergentes. La relación Universidad-Empresa (RUE) es un recurso para construir un pilar fuerte de la innovación. Por lo tanto, es importante establecer una buena relación entre los principales participantes, el emprendedor y el investigador (E-I). Sin embargo, la literatura actual ha ignorado en gran medida el problema del personal involucrado. Para aportar conocimientos sobre este tema, se analizó la brecha existente entre el emprendedor y el investigador (BEI), la importancia de la empatía en la colaboración E-I y los factores que más impactan en esta colaboración desde el punto de vista del emprendedor. El estudio fue exploratorio y transversal y los datos fueron recogidos a través de entrevistas y cuestionarios. La muestra estuvo formada por expertos empresarios con experiencia en proyectos de innovación realizados en colaboración con investigadores. Los resultados muestran que el $70 \%$ de los empresarios entrevistados reconocen la existencia de la BEI. El 87,5\% de los empresarios encuestados considera la empatía como un factor muy significativo para la relación E-I, y hay 32 factores que son más influyentes en esta colaboración.

Palabras clave: Brecha Empresario Investigador (BEI), Innovación Tecnológica, Relaciones Universidad Empresa (RUE), Competitividad, Estrategia, I+D, Transferencia de Tecnología y Conocimiento (TTC)

\section{Introducción}

El pilar de la innovación tecnológica se considera el de mayor valor estratégico para la competitividad y el que tiene más rendimiento sostenido a través del tiempo (World Economic Forum, 2011). La relación universidadempresa (RUE) se reconoce como uno de tres elementos clave para los sistemas de innovación. La importancia de la colaboración de estas dos instituciones se debe a que ambas pueden generar nuevos productos, procesos y conocimientos, lo cual impulsa mejor la investigación y desarrollo (I+D). La colaboración Universidad-Empresa también produce progreso tecnológico (Chen \& Dahlman, 2005) y permite desarrollar un sistema de innovación eficaz en cualquier país del mundo (World Economic Forum, 2011). La colaboración universidad-industria de los países desarrollados y la generación de innovación, están positivamente correlacionadas en el contexto global; esta correlación es muy significativa en el marco europeo (Mercan \& Göktaş, 2011), pero también es muy importante en Estados Unidos y Latinoamerica. 
Las RUE tienen más de tres décadas en estudio y se han analizado diversos temas como: la influencia de la situación geográfica (Muscio, 2013); los canales de colaboración (Fernández, De Souza, da Silva, Suzigan, Chaves, \& Albuquerque, 2010; Bekkers \& Bodas Freitas, 2008; Dutrenit \& Arza, 2010); las oficinas de transferencia tecnologica (OTT) por la importancia para los investigadores en sus actividades empresariales (Göktepe-Hultén, 2010); el comportamiento innovador de las empresas en función de la colaboración universidad-industria (Shichijo, Baba \& Yarime, 2010); la calidad de las relaciones universidad-empresa (Dueñas Quintero \& Duque Oliva, 2015); la madurez de colaboración universidad-industria (Hemmert, Bstieler \& Okamuro, 2014); las prácticas empresariales para las RUE (Montoya \& Aguilar, 2013); el impacto de algunos factores con el éxito y su rendimiento sobre los vínculos universidad-industria. (Plewa, Korff, Baaken \& Macpherson, 2013); la colaboración entre la universidad y las PYMES (Motohashi, 2005; Bjerregaard, 2010), entre otros. Todas las dinámicas de colaboración constituyen una forma de medir los resultados de la investigación y el impacto del quehacer académico de manera cuantitativa, lo cual es un indicador de confianza para la industria (O’Shea, Chugh \& Allen, 2008).

Dichos hallazgos han ayudado a conformar un estado del conocimiento muy extenso, sin embargo, hoy en día la colaboración universidad-industria tiene muchas barreras y pocas explicaciones (Bruneel, D’esteb \& Salter, 2010). Una gran cantidad de los elementos importantes para el intercambio de conocimientos todavía siguen en una "caja negra" (Foss et al., 2009 citado en Ankrah, Burgess, Grimshaw \& Shaw, 2013). También se requiere mejorar la integración de las investigaciones bajo una visión globalizante (Ankrah \& AL-Tabbaa, 2015).

Se percibe, además, que los investigadores académicos necesitan interactuar con los industriales cara-a-cara, con el fin de lograr mayor intercambio de conocimientos y rendimiento científico destacado en el contexto de la innovación tecnológica (Balconi \& Laboranti, 2006). Se conoce también que una alta inteligencia emocional y una alta empatía proporcionan ventajas para la capacidad de liderazgo, la habilidad de enfrentar mejor las tenciones y mejorar la tendencia a ser más innovadores (Humphrey, 2013). No obstante, la literatura actual ha ignorado en gran medida el problema del personal involucrado, sus diferencias y características (Azagra-Caro, 2007).

Uno de los ámbitos escasamente estudiado, es el de la colaboración directa entre el empresario y el investigador (E-I). Este binomio puede situarse como el núcleo de las RUE hablando del aspecto humano y se conoce poco acerca de su día a día. Es necesario profundizar más en el 
estudio del entendimiento y la colaboración entre el académico y el industrial ya que sus relaciones siguen siendo difíciles (Martínez \& Ikertia, 2013).

Además, se requiere analizar la relación del binomio E-I para encontrar cómo aumentar los niveles de confianza mutua y ayudar a reducir el temor de la actuación oportunista (Dodgson, 1993). La confianza aporta seguridad en una actuación justa y consistente, además ayuda en la solución de problemas de manera conjunta (Rempel \& Holmes, 1986; Zaheer et al., 1998; ambos citados por Bruneel, d’Este \& Salter, 2010). Sin embargo, en la realidad no parece haber una relación muy consistente, eficiente y eficaz entre E-I, lo cual repercute en el sistema de innovación tecnológica.

Para representar y analizar este fenómeno de las relaciones entre el empresario y el investigador, en el estudio de Mendez et al. (2016) se presentó el concepto de brecha entre empresario e investigador (BEI) en el contexto específico de ambos actores y desde la perspectiva de relaciones humanas. Entender más al binomio E-I podría ayudar a determinar cuáles son los factores que aportan valor a la relación, cuáles no aportan valor y cuáles obstaculizan la relación para aportar conocimiento al respecto. El presente trabajo analizó la existencia de una brecha entre empresario e investigador (BEI), la importancia de la empatía en la colaboración E-I y los factores que más influyen en esta colaboración desde la perspectiva de los empresarios que participaron en proyectos de I+D y TTC como complemento a la primera parte del estudio realizado por Méndez et al. (2016).

\section{Método}

El estudio tiene un alcance exploratorio, descriptivo y de tipo cualitativo-cuantitativo, transversal. Se aplicó un test a 24 empresarios con experiencia en proyectos de colaboración conjunta de $\mathrm{I}+\mathrm{D}$ con instituciones o centros de investigación públicos y privados. El periodo de tiempo fue de mayo a noviembre del 2016. El estudio se realizó en México.

El procedimiento utilizado, a partir de Méndez et al. (2016), fue el siguiente:

1. Se diseñaron las siguientes preguntas:

La pregunta a) se diseñó para determinar la importancia de la empatía en proyectos conjuntos desde la perspectiva del binomio E-I:

a) ¿La empatía en la relación entre el empresario y el investigador es importante para la participación en proyectos conjuntos de I+D y TTC?

La pregunta b) se dirige a confirmar la BEI según la perspectiva del empresario:

b) ¿Opina Usted que se percibe una brecha entre el empresario y el investigador en el contexto de la I+D y TTC? 
Para captar la contribución de los empresarios sobre nuevos factores que pudieran estar presentes y no se han revelado, se aplicó la pregunta c: c) ¿Qué otro factor o factores considera que afecta la relación empresario y el investigador en el contexto de la TTC e I+D?

2. Para cada uno de los factores de la Tabla 1, se utilizó una escala de Likert de cinco puntos, para conocer la percepcion del empresario sobre los factores de mayor influencia para la colaboración conjunta.

Tabla 1. Factores clasificados y codificados en cognitivos, afectivos y situacionales (CAS) para evaluar la relación empresario-investigador en el contexto de la I+D y TTC

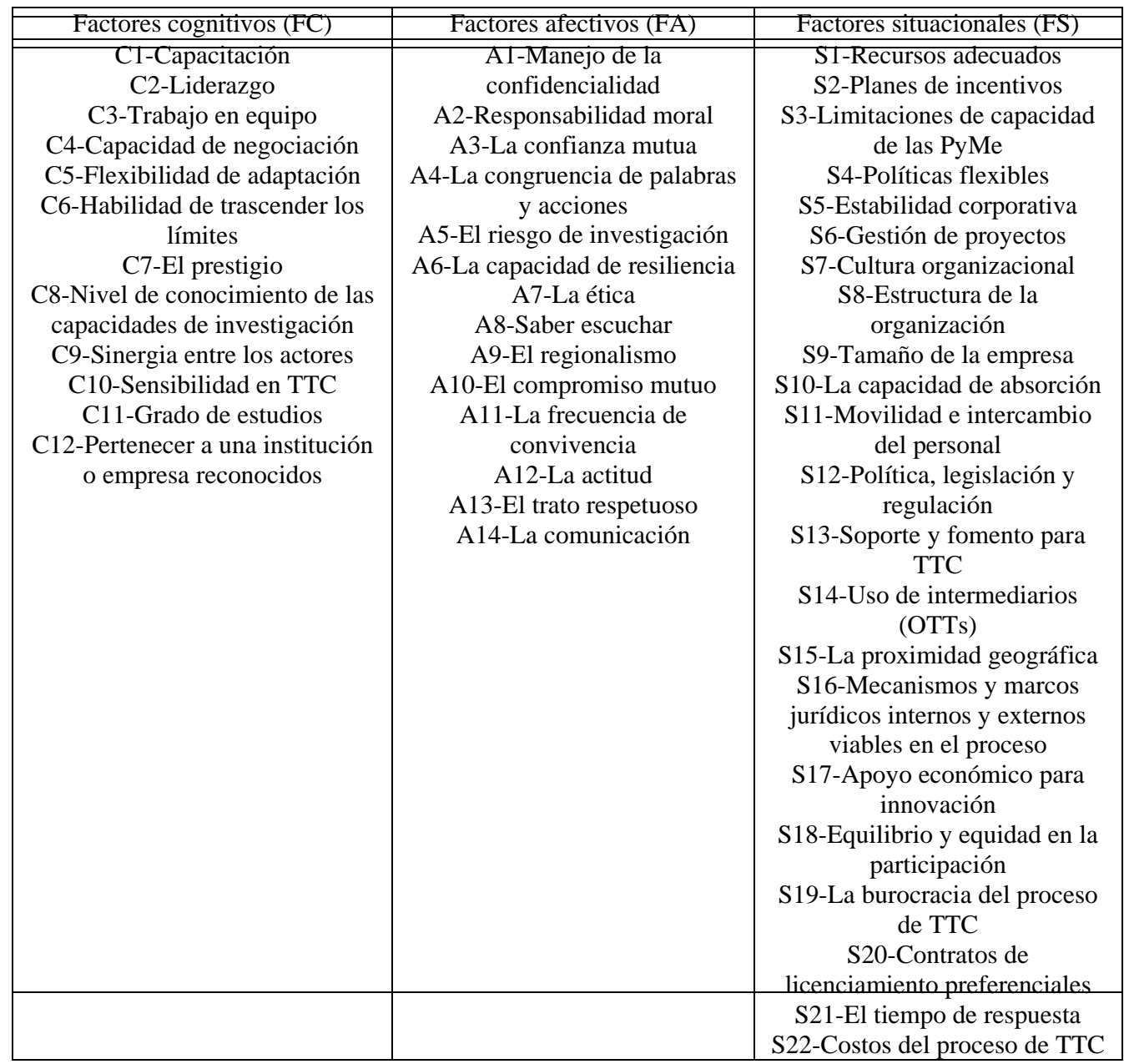

Tomado de (Méndez et al., 2016)

3. Con los tres bloques de factores (CAS) y las tres preguntas descritas arriba, se diseñó el test que se aplicó a los empresarios. 
4. Se realizó una prueba piloto a siete individuos. Se determinó la confiablilidad y consistencia interna del instrumento por el coeficiente Alpha de Cronbach y el paquete estadístico SPSS.

5. Se obtuvo un Alpha de Cronbach $=0.98$ de consistencia interna $\mathrm{y}$ consideró aceptable el instrumento de acuerdo con Campo-Arias y Oviedo (2008).

6. La muestra se obtuvo por conveniencia y oportunidad en base a expertos (Hernández, Fernández \& Baptista, 2010).

7. El instrumento fue aplicado por correo electrónico con reforzamiento vía telefónica y, en los casos, posibles se realizó por entrevista.

8. Se aplicó estadística descriptiva para analizar los datos obtenidos de los test aplicados.

\section{Resultados}

A continuación, se presentan los datos resultantes de la percepción y actitud de los empresarios respecto a la empatía, la BEI y los factores CAS más importantes para su relación.

\section{Sobre la Empatía}

La Gráfica 1 representa las respuestas de los empresarios sobre la importancia de la empatía entre el binomio E-I para su participación en proyectos de colaboración conjunta. Los datos muestran que el $87.5 \%$ de los empresarios que respondieron piensa que la empatía es muy significativa para su participación en proyectos conjuntos de I+D y TTC.

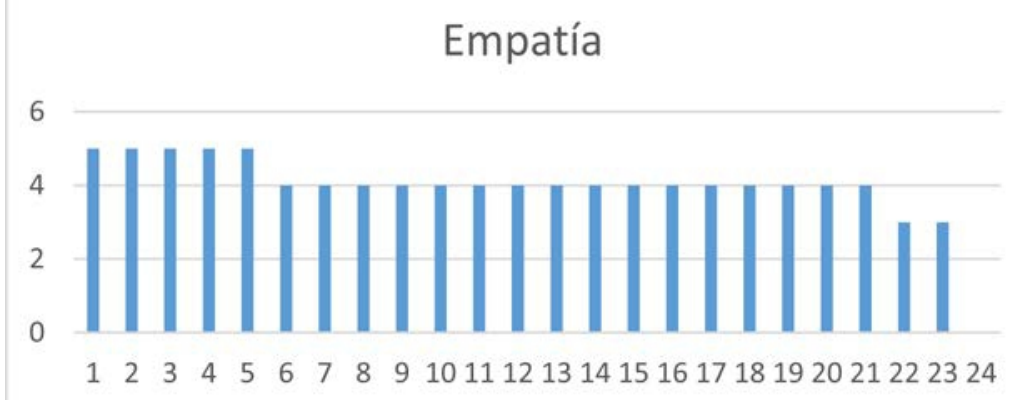

Gráfica 1. Importancia de la empatía según la percepción de los 24 empresarios encuestados. El valor máximo es 5= Lo más importante, el valor mínimo es $1=$ nada importante, $0=$ no contestó.

\section{Sobre la BEI}

La Gráfica 2 muestra los resultados de los empresarios respecto a su percepción sobre la existencia de una brecha entre empresario e investigador. 
Los datos muestran que el $70 \%$ de los empresarios encuestados sí percibe una BEI en proyectos de I+D y TTC.

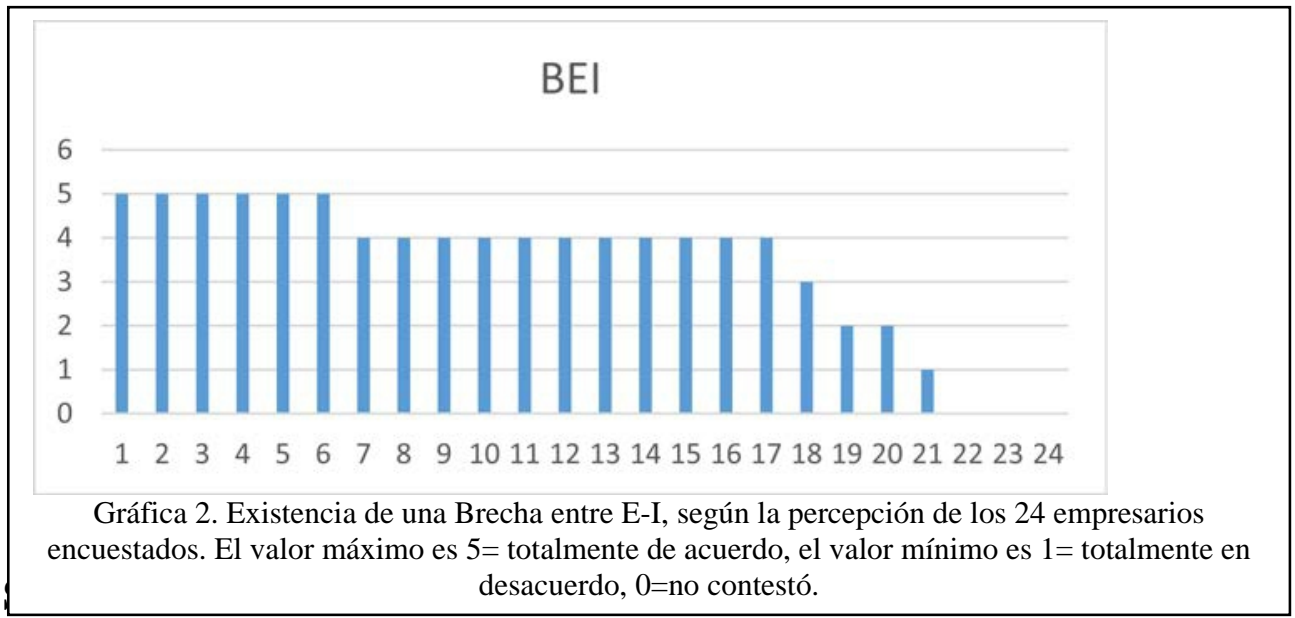

\section{Factores Cognitivos}

A continuación, se presentan los datos obtenidos del análisis de los factores Cognitivos, Afectivos y Situacionales para visualizar cuáles influyen más significativamente en la relación entre E-I, según lo percibe el empresario.

En la gráfica 3 podemos ver que, desde la perspectiva del empresario, el 58\% de los factores cognitivos influyen en la relación E-I.

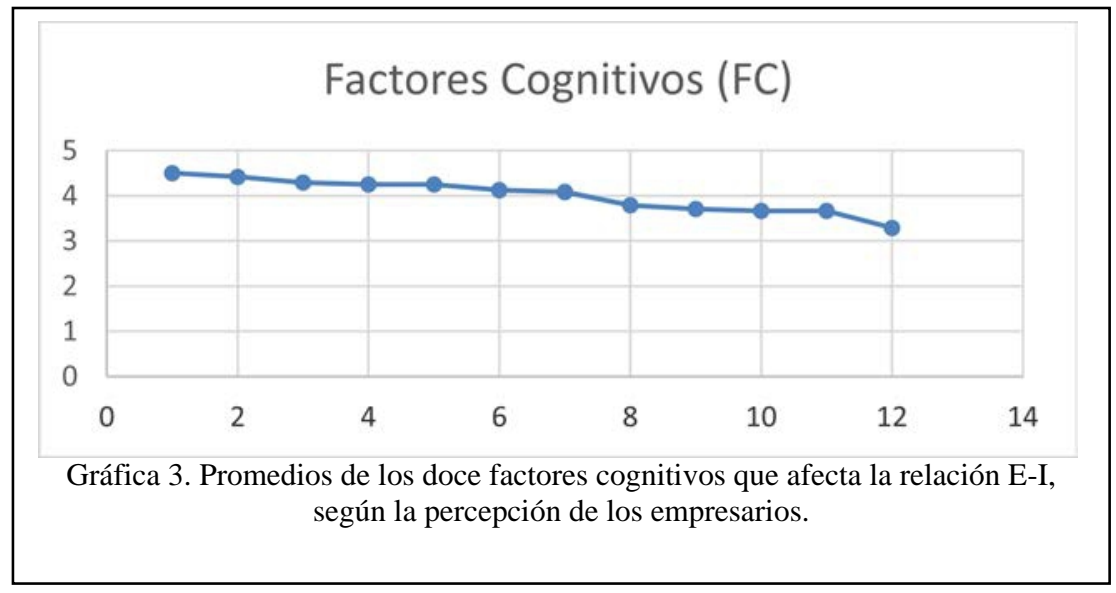

En la gráfica 4 se presentan los datos con mayor puntuación (min=1, máx. $=5$ ) en función del nivel de importancia percibido por los empresarios encuestados sobre los factores cognitivos (FC). 
Se observa que los factores cognitivos más destacados valorados con 5 por los empresarios para la relación E-I fueron: C1, C2, C3, C4, C8, C9 y C12 (ver Tabla 2 para su descripción).

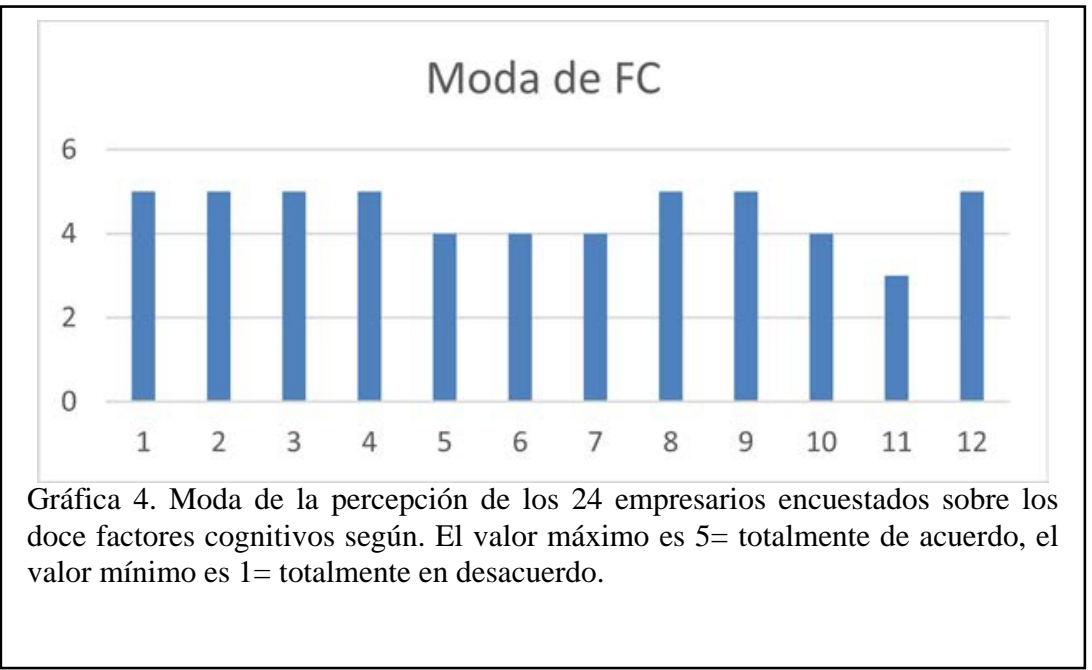

\section{Factores Afectivos}

En la gráfica 5 podemos ver que, desde la perspectiva del empresario, el 86\% de los factores afectivos impactan significativamente en la relación E-I, tomando en cuenta los promedios de los valores asignados por los empresarios iguales o mayores a 4 .

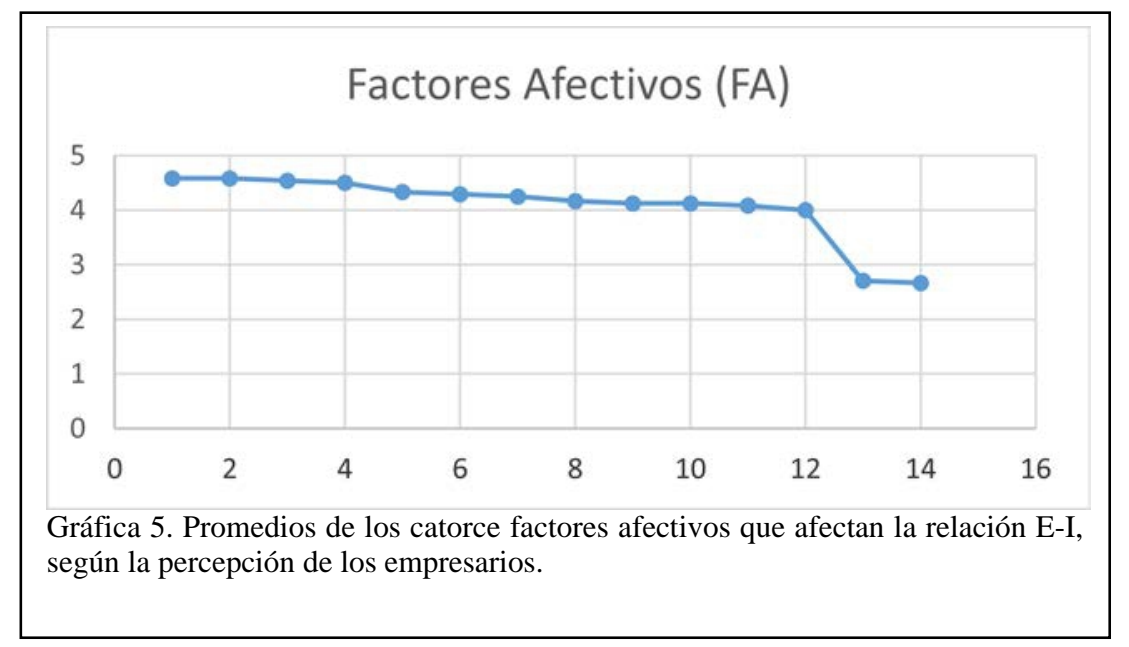

En la gráfica 6 se presentan los factores con mayor puntuación, según los empresarios encuestados.

Se observa que los factores afectivos más destacados por los empresarios para la relación E-I fueron: A1, A2, A3, A4, A7, A8, A10, A11, A12, A13, y A14 (ver Tabla 2 para su descripción). 


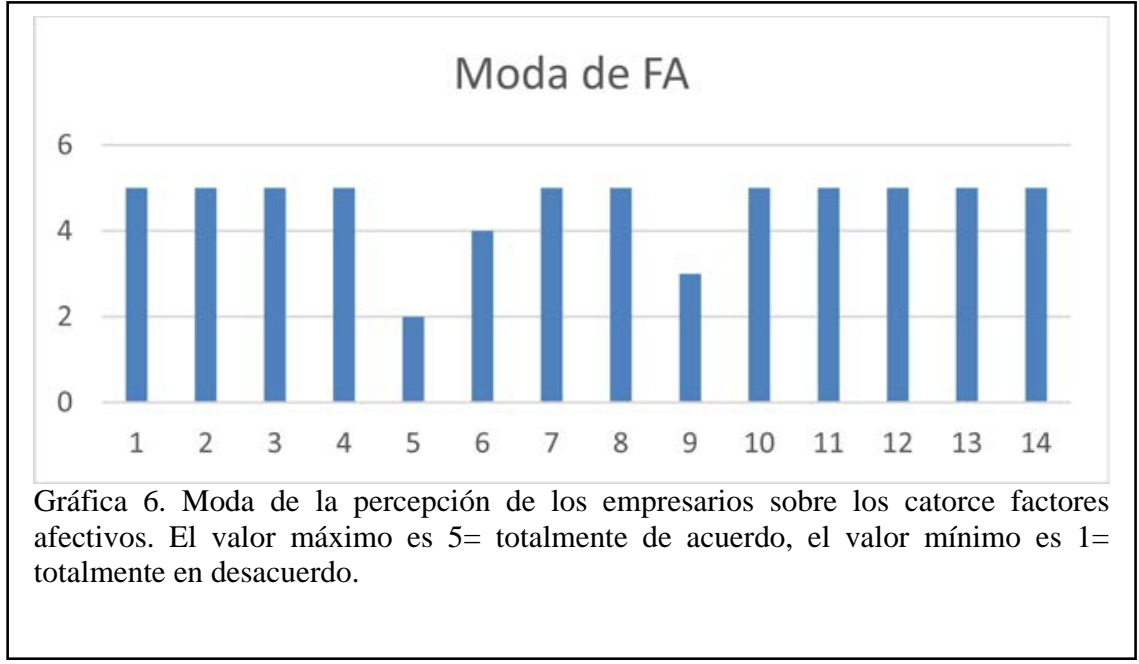

\section{Factores Situacionales Empresarios}

En la gráfica 7 se presenta la perspectiva del empresario sobre los Factores Situacionales. Los datos muestran que el $54 \%$ de dichos factores influyen en la relación E-I.

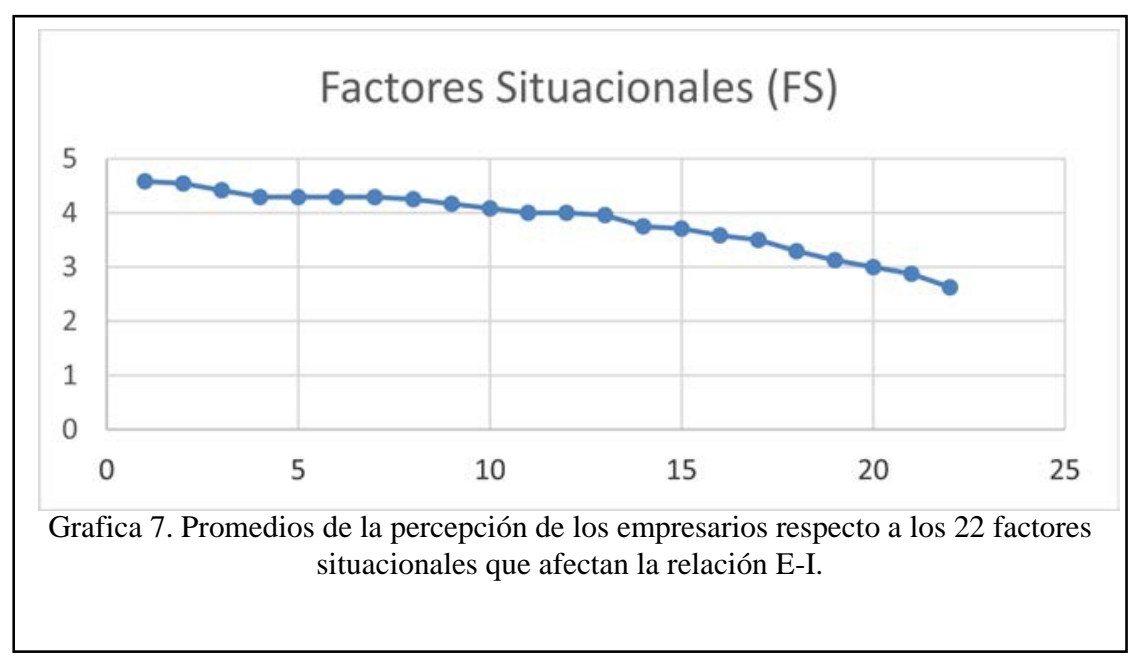


En la gráfica 8 se presentan los datos con mayor puntuación (min=1, máx.=5) en función del nivel de importancia percibido por los encuestados. Los factores situacionales más destacados por los empresarios para la relación E-I fueron: S1, S2, S3, S4, S5, S6, S7, S8, S10, S13, S17, S18, S19, y S21 (ver Tabla 2 para su descripción).

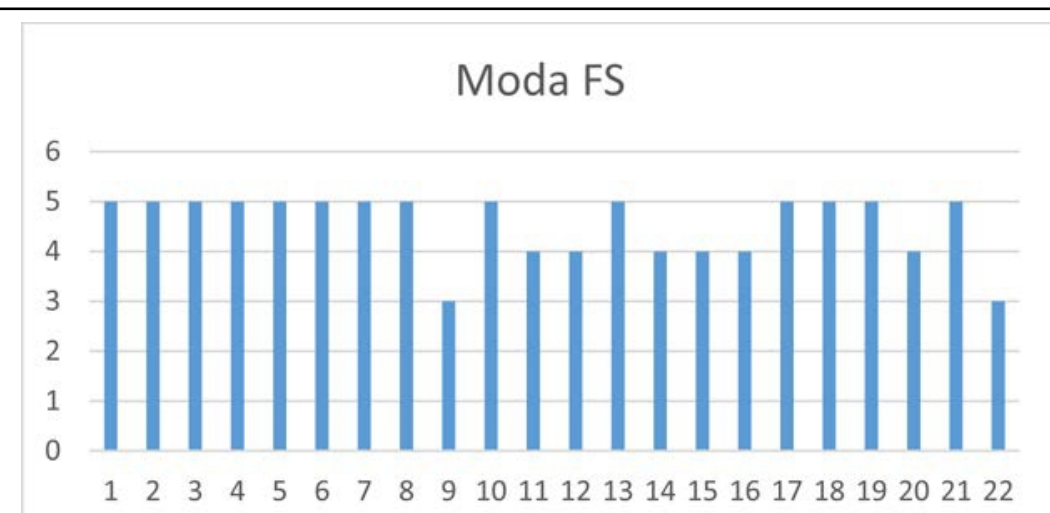

Gráfica 8. Moda de la percepción sobre los factores situacionales según los empresarios encuestados. El valor máximo es $5=$ totalmente de acuerdo, el valor mínimo es $1=$ totalmente en desacuerdo.

Los factores CAS que más influyen en el binomio E-I de acuerdo a la percepción de los empresarios encuestados se resumen en la Tabla 2.

Tabla 2. Factores CAS que mas influyen en el binomio E-I de acuerdo a la opinión del empresario

\begin{tabular}{|c|c|}
\hline Tipo de factor & Descripción de los factores \\
\hline \multirow{4}{*}{ Cognitivo } & C1-Capacitación \\
& C2-Liderazgo \\
& C3-Trabajo en equipo \\
& C4-Capacidad de negociación \\
& C8-Nivel de conocimiento de las capacidades de investigación \\
C9-Sinergia entre los actores \\
C12-Pertenecer a una institución empresa reconocidos \\
\hline Afectivo & A1-Manejo de la confidencialidad \\
& A2-Responsabilidad moral \\
& A3-La confianza mutua \\
& A4-La congruencia de palabras y acciones \\
& A7-La ética \\
& A8-Saber escuchar \\
& A10-El compromiso mutuo \\
& A11-La frecuencia de convivencia \\
A12-La actitud \\
A13-El trato respetuoso \\
A14-Comunicación \\
\hline Situacional & S1-Recursos adecuados \\
& S2-Planes de incentivos \\
& S4-Políticas flexibles \\
& S5-Estabilidad institucional \\
\hline
\end{tabular}




\begin{tabular}{|c|c|}
\hline & S6-Gestión de proyectos \\
S7-Cultura organizacional \\
S8-Estructura de la institución \\
S10-La capacidad de absorción \\
S13-Soporte y fomento para TTC \\
S17-Apoyo económico para innovación \\
S18-Equilibrio y equidad en la participación \\
S19-La burocracia del proceso de TTC \\
S21-El tiempo de respuesta \\
\hline
\end{tabular}

Otros factores que se detectaron a través de las aportaciones de los empresarios según su experiencia en la colaboración conjunta en proyectos de I+D se presentan en la tabla 3.

Tabla 3. Otros factores que afectan la colaboración en proyectos conjuntos de I+D y TTC desde la perspectiva de los empresarios

\begin{tabular}{|c|c|}
\hline Factores aportados & Descripción \\
\hline Factores cognitivos & $\begin{array}{c}\text {-Conocimientos, aptitudes y lenguaje } \\
\text {-Falta de cultura empresarial en los investigadores } \\
\text {-Escasa sinergia para la retroalimentación entre E-I } \\
\text { investigador } \\
\text {-Urgencia de Tiempos de entrega distintos para el empresario y el } \\
\text {-Falta de cultura de TTC } \\
\text {-Interactuar cara a cara con el empresario y el cliente del proyecto } \\
\text {-Monitoreo y actualización de las tendencias del mercado por parte de la } \\
\text { institución } \\
\text {-Necesidad de acompañamiento del proyecto por un investigador } \\
\text { especializado en el tema desde el inicio } \\
\text {-No se da en realidad el trabajo en equipo } \\
\text {-Proyectos motivados por una necesidad real } \\
\text { emprendedor } \\
\text {-Tiempos de los académicos diferentes a los del empresario o } \\
\text {-El investigador no sabe vender su idea ni mostrar claramente sus } \\
\text { expectativas, valor agregado y método de trabajo } \\
\text {-Se necesita mayor profesionalismo en la investigación y la contratación } \\
\text { para hacer investigación } \\
\text {-Tiempos y costos de E-I desfasados con el mercado } \\
\text {-Diferente conceptualización del tiempo de las actividades en academia e } \\
\text { investigación y la iniciativa privada (IP) } \\
\text {-Objetivos de la academia distintos a los de la IP (artículos, horas de } \\
\text { proyectos, etc. vs mejoras tangibles (físicas) en productos y procesos }\end{array}$ \\
\hline Factores afectivos & $\begin{array}{c}\text {-Cumplir con los compromisos y acuerdos } \\
\text {-Falta de compromiso con el proyecto } \\
\text {-Sensibilidad de la Universidad con el empresario } \\
\text {-Resistencia del investigador a salir de su círculo de confort } \\
\text {-Preferencia por actividades sencillas y reticencia por las complicadas por } \\
\text { algunos investigadores } \\
\text {-Falta de responsabilidad de la IES ante el proyecto } \\
\text {-Poco empoderamiento del investigador } \\
\text { - Falta una relación más estrecha con el investigador } \\
\text {-Responsabilidad conjunta } \\
\text {-El factor psicológico entre el empresario y el investigador }\end{array}$ \\
\hline Factores situacionales & $\begin{array}{c}\text {-Largos tiempos de respuesta } \\
\text {-Costos no siempre justificables } \\
\text {-Complicado proceso de gestión } \\
\text {-No hay programas permanentes de colaboración E-I }\end{array}$ \\
\hline
\end{tabular}




\begin{tabular}{|c|c|}
\hline -Tiempos de respuesta de las OTT \\
-La burocracia de la universidad \\
-Falta de políticas para asegurar la continuidad de las actividades cuando \\
hay huelgas, paros o bloqueos inesperados que entorpecen o impiden el \\
seguimiento del proyecto \\
-La normatividad de los procesos internos para la adquisición de equipos y \\
materiales de los proyectos conjuntos. \\
-La organización y gestión interna \\
-Falta de fiscalización técnica por expertos en el tema del proyecto \\
-Falta de normas acordes al proyecto \\
-Elevar el valor de las actividades de I+D para el investigador \\
-La organización y gestión interna \\
-Burocracia, lentitud, de la IES ante el proyecto \\
-Reglamentación interna \\
-Falta de estándares de vinculación \\
-El intermediario por parte de la empresa (oficina o despacho de \\
vinculación) entre E-I \\
-Flexibilidad en el uso del recurso destinado al proyecto \\
-La restricción de tiempos de CONACYT \\
-Burocracia interna de universidad
\end{tabular}

\section{Conclusion y Recomendaciones}

Las relaciones entre la universidad y la empresa se han estudiado en diferentes líneas y con distintos enfoques. Entre todas las investigaciones revisadas para este estudio se encontró que no se había estudiado al empresario y al investigador con un enfoque humano y con la perspectiva de ambos sobre los mismos elementos, pensando en que el binomio es el núcleo de las relaciones universidad empresa, hasta el estudio de Méndez et al. (2016). Al sumar esta segunda parte del estudio de la BEI se ha encontrado empíricamente que ambos (E-I), están siendo influenciados por los tres factores CAS (cognitivos, afectivos y situacionales) lo cual altera la relación armónica con su contraparte.

Con los resultados obtenidos en este estudio también se logró encontrar que el $87.5 \%$ de los empresarios encuestados, piensa que la empatía es muy significativa para su participación en proyectos conjuntos de I+D y TTC. Esto refuerza lo encontrado por Méndez et al. (2016) sobre los investigadores quienes también manifestaron que la empatía es muy significativa.

El otro resultado obtenido confirma que existe una brecha entre el empresario y el investigador (BEI), ya que el 70\% de los empresarios encuestados manifiesta que percibe dicha brecha. En la investigación de Méndez et al. (2016) se encontró que los investigadores perciben también dicha brecha con un $75 \%$ de acuerdo. Es posible entonces establecer que en realidad existe la BEI y que se puede adoptar como una nueva línea de investigación para aumentar el conocimiento respecto a la realidad de la relación directa del binomio E-I, como una estrategia de entendimiento y mejora de las relaciones universidad empresa (RUE). 
Además, los resultados indican que los empresarios se ven más influenciados por el 66\% de los factores conocidos (32 de 48) para la relación E-I en comparación con la opinión de los investigadores, que manifiestan que solo el 17\% (8 de 48) influye más significativamente en la relación entre el empresario y el investigador (Méndez et al., 2016).

Esta diferencia en el impacto complementa el argumento de la existencia de una BEI. Y muestra que los intereses y las preocupaciones de ambos actores tienen pesos diferentes y además respalda la idea de que es necesario estudiar más las capacidades y características personal involucrado con un enfoque más humano como lo sugería Azagra-Caro (2007).

Los factores que más impacto tienen para los empresarios deberían ser de interés para los directivos y el gobierno en pos de una mejora del sistema de innovación, dado que así, esfuerzos por mejorar las relaciones universidad empresa serían más eficaces.

Al reducir la BEI se estaría avanzando en la consolidación del sistema de innovación tecnológica del país.

\section{References:}

1. Ankrah S., \& AL-Tabbaa O. (2015). Universities-industry collaboration: A systematic review. Scandinavian Journal of Management, 31, 387-408.

2. Ankrah, S. N., Burgess, T. F., Grimshaw, P. \& Shaw, N. E. (2013). Asking both university and industry actors about their engagement in knowledge transfer: What single-group studies of motives omit. Technovation, 33, 50-65.

3. Azagra-Caro, J. M. (2007). What type of faculty member interacts with what type of firm? Some reasons for the delocalisation of university - industry interaction. Technovation, 27, 704-715.

4. Balconi, M. \& Laboranti, A. (2006). University-industry interactions in applied research: The case of microelectronics. Research Policy, 35 (10), 1616-1630.

5. Bekkers, R. \& Bodas Freitas, I. (2008). Analysing knowledge transfer channels between universities and industry: To what degree do sectors also matter? Research Policy, 37, 1837-1853.

6. Bruneel, J., d'Este, P. \& Salter, A. (2010). Investigating the factors that diminish the barriers to university-industry collaboration. Research Policy, 39 (7), 858-868.

7. Campo-Arias, A. \& Oviedo, H. (2008). Psychometric properties of a scale: internal consistency. Revista de Salud Pública. 10 (5), 831-839.

8. Chen, D.H. \& Dahlman, C.J. (2005). The knowledge economy, the KAM methodology, and World Bank operations. World Bank 
Institute. Working Paper.Technological collaboration in industry: strategy, policy, and internationalization in innovation. Routledge.

9. Dutrenit, G. \& Arza, V. (2010). Channels and benefits of interactions between public research organizations and industry: comparing four Latin American countries. Science and Public Policy, 37 pp. 541553.

10. Dueñas Quintero, D. M., \& Duque Oliva, E. J. (2015). Calidad de las Relaciones Universidad-Empresa: un análisis desde el enfoque de marketing relacional en Boyacá. Pensamiento \& Gestión, (38), 147175.

11. Fernández, A. C., De Souza, B. C., da Silva, A. S., Suzigan, W., Chaves, C. V. \& Albuquerque, E. (2010). Academy-industry links in Brazil: evidence about channels and benefits for firms and researchers. Science and Public Policy, 37 (7), 485-498.

12. Göktepe-Hultén, D. (2010). University-industry technology transfer: who needs TTOs? International journal of technology transfer and commercialization, 9 (1-2), 40-52.

13. Hemmert, M., Bstieler, L. \& Okamuro, H. (2014). Bridging the cultural divide: Trust formation in university-industry research collaborations in the US, Japan, and South Korea. Technovation, 34, 605-616.

14. Hernández Sampieri, R., Fernandez Collado, C. \& Baptista Lucio, P. (2010). Metodología de la investigación. México: McGraw-Hill/ Interamericana editores S.A. de C.V.

15. Humphrey, R. H. (2013). The benefits of emotional intelligence and empathy to entrepreneurship. Entrepreneurship Research Journal, 3 (3), 287-294.

16. Martínez, R. \& Ikertia, F. (2013). La relación universidad-empresa en bizkaia: Un enfoque cualitativo y propuesta de mejora. Revista EAN, (61), 123-126.

17. Méndez Peón, C. Á., Herrera Avilés, M., Toriz Palacios, A., \& González Pérez, M. (2016). Estudio De La Brecha EmpresarioInvestigador. La Percepción Del Investigador. European Scientific Journal, ESJ, 12 (28).

18. Mercan, B., \& Göktaş, D. (2011). Components of innovation ecosystems: a cross-country study. International Research Journal of Finance and Economics, 76, 102-112.

19. Montoya Ríos, F. J. \& Aguilar Zambrano, J. J. (2013). La Relación Universidad-Empresa en las Prácticas Empresariales: un Modelo Conceptual desde las Técnicas de Generación de Ideas. Journal of technology management \& innovation, 8, 47-47. 
20. Muscio, A. (2013). University—industry linkages: What are the determinants of distance in collaborations? Papers in Regional Science, 92, 715-739.

21. O’Shea, R. P., Chugh, H., \& Allen, T. J. (2008). Determinants and consequences of university spinoff activity: a conceptual framework. The Journal of Technology Transfer, 33 (6), 653-666.

22. Plewa, C., Korff, N., Baaken, T. \& Macpherson, G. (2013). University-industry linkage evolution: An empirical investigation of relational success factors. R\&D Management, 43, 365-380.

23. Shichijo, N., Baba, Y. \& Yarime, M. (2010). Sources of success in advanced materials innovation: The role of "core researchers" in university-industry collaboration In Japan. International Journal of Innovation Management, 14, 201-219.

24. World Economic Forum (2011). The global competitiveness report 2011-2012. Geneva: WEF. 